\section{Triptan Quality Limits}

Dear Editor,

I am writing in response to Dr. Culley's and Dr. Wanovich's article in JMCP November/December 2001 regarding triptan limits. ${ }^{1}$ While I commend their efforts to seek answers on how to judiciously utilize these medications, I have some rather fundamental questions:

1. How many of the 105 study patients who were denied a triptan either remained at home, skipped work, missed school, or did not perform life's routine tasks but rather suffered through a migraine attack?

2. How many people actually had medication-induced headache (MIH) as opposed to poorly controlled migraine?

3. Who pays the "dispensing pharmacist" to call the MCO?

4. How many patients paid for a triptan out of their own pocket?

5. Does decreased cost of care equate to improved and/or quality care?

Taking a triptan more often than prescribed is not a natural act. Frustrated patients do this because migraine tends to be an insidious, forever worsening, condition; yet patients are rarely offered adequate treatment and/or proper medication counseling. ${ }^{2,3}$ The multiple barriers migraine individuals must overcome to find effective treatment have been documented. ${ }^{4,5}$ In my experience working at one of this country's two tertiary headache clinics' hospital units (where MIH is a leading admission diagnosis), patients self-discover relief with daily or near-daily triptan use. Once this discovery is made, it is difficult to convince these skeptical patients to do otherwise (I know because I try every day) since they have already endured years and even decades of countless inappropriate and/or ineffective therapies.

Far too many migraine patients ultimately succumb to the notion that modern medical science cannot help them. ${ }^{6}$ Patients don't seek help at the emergency room or other points of the health care system because those places have rarely helped in the past. This may partly or wholly explain why this study's patients did not utilize other health services. People just give up and suffer. Where are the outcomes regarding these patients, especially the 105 denied triptan prescription refills?

As the authors point out, over-use of triptans can cause MIH, but they do not say how many individuals in their study actually had this condition. Clinicians who work exclusively with headache patients agree, in principle, that frequent triptan use should be discouraged. However, how frequent is too frequent? Nobody knows for sure. Case reports, clinical experience, and other anecdotal evidence have shown that daily triptan use is not always detrimental and may in fact be beneficial for select patients, for up to three years. ${ }^{7}$ Additionally, we safely and effectively prescribe short courses (3-5 days) of daily triptans for predictable migraine situations such as menstrual migraine. The U.S. Headache Consortium's evidence-based migraine treatment guidelines, the most authoritative document on migraine therapy, recommends that acute medications such as triptans not be used more than two days per week. ${ }^{8}$ However, they also note that this recommendation is not absolute and further research is needed.

In the next few months frovatriptan will become available on the U.S. market. ${ }^{9}$ This drug's half-life is more than 24 hours, far exceeding the half-lives of existing triptans. Some headache specialists speculate that this long half-life may yield a triptan that can, and should, be utilized on a daily basis for chronic headaches. Also, I know from personal communications that there are ongoing controlled trials of daily use with already-marketed triptans. I eagerly await the results of these researchers' efforts.

The study did discuss a mechanism for patients to exceed triptan limits by having the "dispensing pharmacist" call the MCO in order to seek a "justified" (not defined) reason. However, is creating additional work for an already overwhelmed retail pharmacist an ideal intervention? Where are the costs of these phone calls reported? Being placed on hold after calling an MCO for an hour or more happens on an alarmingly regular basis. And how many study patients, once denied, simply opened their wallets and got the triptan drug anyway? The study's results do not comment on this. My patients tell me they routinely do this.

Under-utilization of migraine prophylactic drugs is seen frequently in practice. The study's results show that use of prophylactic drugs increased, but only slightly, 22,433 versus 23,201 prescriptions filled. This meager increase does not demonstrate that people who would benefit from prophylactic drugs received them because of enforcing triptan limits.

I also strongly question the authors' expectations that "limitations on the triptans could cause an increase in the use of acute pain medications (analgesics, etc.)." Step care (prescribing a nonspecific drug such as an analgesic and progressing towards migraine-specific drugs such as triptans only after nonspecific drugs fail) is the most commonly utilized migraine treatment strategy in this country, even though it has been shown inferior to stratified care. ${ }^{10}$ Thus, for the comparatively few migraine patients who are prescribed a triptan, the majority have already tried and failed analgesics. ${ }^{11}$ Most patients and doctors are unwilling to go back to unsuccessful treatments.

I give continuing education lectures to pharmacists about migraines. It is obvious that migraine and medication-induced headaches are illnesses surrounded by mystery, misunderstanding, and improper treatment. Lack of education may be one reason for this confusion. Research shows that the typical pharmacy student receives only one contact hour of classroom education per year regarding headache disorders, and only two schools in the entire country offer clerkships dedicated exclusively to headaches. ${ }^{12}$

The American Migraine II study shows migraine headaches profoundly affect the lives of at least 28 million people (not 23 million as reported by the authors), only $48 \%$ of whom are diagnosed by a physician. ${ }^{13}$ Worse, up to $82 \%$ of people who present to tertiary headache centers are experiencing $\mathrm{MIH} .{ }^{14,15}$ The leading cause of chronic daily headaches (CDH) is $\mathrm{MIH}$, and $\mathrm{CDH}$ consume a disproportionate amount of all the resources devoted to treatment of primary headache disorders. ${ }^{16}$

While the authors noted a figure of $\$ 17.2$ billion, this number should be quantified to illustrate that up to $\$ 17.2$ billion annually 
is lost to decreased productivity. The direct medical costs of migraine have been calculated at $\$ 1$ billion annually.13 Thus disability, not direct care costs (i.e., triptans), imposes the greatest economic burden. Effective migraine therapies must be aimed at reducing disability, not merely limiting the costs of drugs. Get people back to work, back to school, back to life's daily tasks, and you will save society a lot of money.

As a result of their acquisition cost and often inappropriate utilization, triptans are targets for MCO scrutiny. As with any discussion of pharmacoeconomics the definition of "cost" must be explained. Obviously if a patient does not use a triptan the MCO has no cost. However, the Panel on Cost-Effectiveness in Health and Medicine endorses a society perspective.$^{18}$ What is the cost to society of a patient suffering at home (thus not at work) with a migraine? Also the poorly treated patient, who has already paid an insurance premium, pays yet again in terms of pain, disability, and actual dollars. Denying people access to care to reduce expenses does not automatically equate to quality care. Such an approach may actually raise costs for patients and society as a whole.

Rather than limiting triptan access, why not attempt to direct these patients to appropriate therapy, i.e., find out why they are taking frequent triptans in the first place and fix THAT? There are validated tools including the migraine-disability assessment questionnaire (MIDAS) and the Headache Impact Test (HIT-6) that can help quantify the onus of headaches on patients' lives as well as guide treatment. ${ }^{19,20}$ The Consortium's evidence-based guidelines advocate stratified care, not step care, as the premium approach to migraine therapy. There are over 200 specialized headache centers in this country where patients can be referred. Get the poorly managed patient to a knowledgeable clinician who will properly prescribe demonstrated effective drugs and the resulting migraine expenses will not be excessive.

Pharmacists are well positioned to make positive changes for migraine sufferers. A recommendation of an OTC "headache product" is the number one OTC product suggestion pharmacists perform, occurring over 53,000 times per day. ${ }^{21}$ Also, as illustrated by the study, MCOs employ pharmacists who create policies that can have a positive (or negative) impact on patients.

The results of this study do not show that triptan limits, though well-intentioned, were beneficial for patients. The results only demonstrate a benefit for "the bottom line." Triptan limits are just one more barrier for patients to find effective help. Since most patients have already dealt with numerous barriers before, the poorly managed, defeated, and in this study unreported migraine 
patients will quietly tolerate yet another barrier.

I speak on their behalf.

Richard Wenzel, Pharm.D., Diamond Headache Clinic Inpatient Unit, National Headache Foundation, Member and Therapeutic Guide Committee Member; American Headache Society - Member; Adjunct Professor, University of Illinois-Chicago, College of Pharmacy E-mail: rwenz@hotmail.com

\section{REFERENCES}

1. Culley EJ, Wanovich RT. Medical and pharmacy cost and utilization outcomes of a quantity limit on the 5-HTl agonists (triptans) by a Managed Care Organization. J Man Care Pharm 2001;7(6):468-75.

2. Lipton, RB, Cady RK, Stewar WF, Wilks K, Hall C. Diagnostic lessons from the Spectrum study, Neurology 2002;58(Suppl):S27-S31.

3. Cady RK, Schreiber CP. Sinus headache or migraine? Neurology 2002;58(suppl 6):S10-S14.

4. Lipton RB, et al. Migraine: Identifying and removing barriers to care. Neurology 1994;44(suppl 4): S63-S68

5. Edmeads J, Laines M, Brandes JL, Schoenen J, Freitag F. Potential of the Migraine Disability Assessment Questionnaire as a public health initiative and in clinical practice. Neurology 2001;56(suppl 1):S29-S34.

6. Lipton RB, Silberstein SD. The role of headache-related disability in migraine management. Neurology 2001;56(Suppl 1):S35-S42.

7. Evan RW, Robbins L. Daily triptans for headache. Headache 2001;41:907-09.

8. Silberstein SD (for the U.S. Headache Consortium). Practice parameter: Evidence-based guidelines for migraine headache. Neurology 2000;55:754-62.

9. Silberstein, SD. Pharmacological profile and clinical characteristics of frovatrip$\tan$ in the acute treatment of migraine: Introduction. Headache 2002;42(suppl 2):S45-S46.

10. Lipton RB, Stewart WF, Stone AM, Lainez MJA, Sawyer JPC. Stratified care versus step care strategies for migraine. JAMA 2000;284;2599-2605.

11. Stang PE, Osterhaus JT, Celentano DD. Migraine: Patterns of healthcare use. Neurology 1994:44(suppl 4):S47-S54.

12. Wenzel RG, Neidich MR. Headache education in Colleges of Pharmacy. Annals of Pharmacotherapy 2002; In press.

13. Lipton RB, Diamond S, Reed M, Stewart WF. The burden of migraine: Compelling insights from the American Migraine Studies. Consultant 2000;40(11):S8-S12.

14. Zed J, Loewen PS, Robinson G. Medication-induced headache: Overview and systematic review of therapeutic approaches. Ann Pharmacother 1999;33:61-72.

15. Wenzel, RG, Sarvis CA. Do butalbital-containing products have a role in the management of migraine? Pharmacotherapy 2002;22(8):In press.

16. Diamond M. Health Conditions Survey. Paper presented at: The 14th Annual Practicing Physician's Approach to the Difficult Headache Patient; February 13, 2001; Rancho Mirage, California.

17. Kaniecki RG. The impact of migraine: How this disorder affects us all. Consultant 2000; 40(11):S25-S28.

18. Lofland JH, Kim SS, Batenhorst, AS, et al. Cost-effectiveness and cost-benefit of sumatriptan in patients with migraine. Mayo Clin Proc 2001;76:1093-1101.

19. www.headachetest.com

20. www.midas-migraine.net.

21. (No author listed). OTC Products: A study of pharmacists' recommendations. Pharmacy Times. September 2001;(suppl):10-30.

\section{A Neurologist's Perspective on Quantity Limits}

Dear Editor,

Perhaps we could put them in barrels - to be placed strategically on street corners. Those who pass may take what they "need." All would be happy.

Shift, momentarily, to narcotics. If it is true that we, doctors, do patients a disservice by restraining our use of narcotics, perhaps the responsibility should be lifted from physicians. Perhaps narcotics, which are largely inexpensive to manufacture, should be made widely available. Thus none would "go hungry."

Or is it that we limit such access for another reason? Is it that we have found a "dark side" to access without restraint? Is it that limitation is an act of kindness?

Narcotics are clearly acknowledged to have "a dark side." Used wisely and appropriately, with reasonable restraint, they serve the patient-relieving both pain and anguish. Yet they may also enslave the patient. When "pain" is a metaphor for anguish, and when narcotics are used to escape angst (a form of "pain" with similar vocabulary), then narcotics enslave. They serve not to build effectiveness nor to capture capacity, but to encourage dissolution. Thus, sanguine medical care calls upon physicians to provide narcotics with restraint-recognizing that correction of the illness is really the ultimate goal.

In this same vein we must, in my opinion, use triptans with recognition that they produce a temporary relief, and not a correction of the underlying proclivity to migraine. If they are used wisely, and with restraint, they serve the patient-providing muchneeded relief with comparative safety. Yet if headaches are frequent and triptans are used only as a temporary escape, then surely the illness shall exact a greater cost. And this is not the goal of optimal medical care.

We may debate what we would call "reasonable" or "excessive" use of triptans. However, for me the issue is simple. I simply ask myself, "Is my prescription of triptans resulting in a maximally functioning human being?" If I encourage the patient to turn repeatedly and frequently to triptans I believe I am only serving to encourage the enslavement of the patient. Alternatively, if I provide these valuable medications for the patient to use occasionally, with other agents serving to control an ongoing headache tendency, then I believe I am serving the patient's overall best interests.

John P. Barbuto, M.D., Medical Director, Outpatient Neurology, Healthsouth Rehabilitation Hospital, Sandy, Utah E-mail: doctorbarbuto@aol.com

\section{The Authors' Respond}

Dear Dr. Wenzel,

We are responding to your comments in your letter to the editor of JMCP. We thank you for your comments and will address as many of your issues as possible. The objective of our study was to examine the overall impact of the quantity level limit on all triptans users in our managed care lines of business (nearly 12,000 members), not specifically the 105 patients that were denied additional quantities of medication. ${ }^{1}$ We would like to clarify that the members who were denied additional quantities of medication were still eligible for the 
amounts listed in the article and we would not deny a refill that was within those limits. We are concerned about our members and any adverse impact this quantity limit may have had. There are questions that remain regarding the impact on productivity and quality of life that our study does not address. To date, there is a paucity of literature that adequately addresses this subject, to which there are no adequate answers. Although the quality of life issues are certainly important, they are far outside the scope of our study.

We agree that people who suffer from migraines have a devastating disease and there is no one-size-fits-all way to manage them. That is precisely the reason we created an exception process. We realize there are members in our population that experience more frequent migraines than most others. Please keep in mind that in our study, 56\% of all requests for additional quantities of triptans were approved; in December 2001, our approval rate was 88\%. Interestingly $18 \%$ of all requests required a clinical review. For the remaining $82 \%$ of calls that were handled, no additional quantities were requested, typically because the physician agreed the edit limits were sufficient for their patients. This suggests that many members were obtaining prescriptions for large quantities, not because they needed to, rather because they could. Stockpiling, prescription sharing, and other fraudulent practices happen on a daily basis and only serve to increase the cost of health care for all insured people.

As you stated, rebound or medication-induced headaches (MIH) are a serious problem. In your reference, $82 \%$ of people who present to a tertiary headache center experience $\mathrm{MIH} .{ }^{2}$ Clearly this illustrates that physicians, pharmacists, and patients are having difficulty with the proper use of these mediations and the management of this debilitating condition. By implementing a quantity limit, we hoped to identify these highest uses and hopefully prevent our members from ever reaching that state of poor migraine control. Also, because of our exception review process, we frequently had discussions with physicians about their patients who use more than our limits and made exceptions for those members while also encouraging the use of prophylactic medications and referrals to specialists when necessary.

There were many cases of medication-induced headache that were brought to our attention, but we did not specifically track individual cases. In our population, we discovered cases where physicians did not know how many tablets their patients were taking on a monthly basis, patients obtained prescriptions from multiple physicians and, in some cases, physicians requested quantities sufficient for continual twice-daily dosing of the triptans. Continuing to pay for this daily use of medication does not encourage the patient to break the rebound cycle. We work very closely with our physician community and neurologists who specialize in headache and migraine therapy. We are well aware of the problems patients encounter when they are experiencing a cycle of medicationinduced headaches/migraines.

We agree that one of the limitations of our study was not being able to account for therapies that did not create an insurance claim. We did not have the necessary resources available to adequately study this issue to determine its frequency and impact.

Regarding your question about our choosing to analyze the use of acute pain medication (analgesics), our claims data indicated that most of our triptan-using population (over 92\%) are also currently taking analgesics. You cite a study in your letter that suggested that only a few migraineurs are actually taking triptans. ${ }^{3}$ At that point (1994), the triptans as a class were relatively new and sumatriptan was the only triptan available on the market. Prescribing habits have changed since the introduction of four (soon to be five) additional drugs in this class. We used our claims history to analyze the utilization of other analgesics, realizing that the quantity limit on the triptans may have encouraged the use of other pain medications. However, as stated in our study, this was not the case.

Lastly, we never would want to be the cause of any harm to our members. We too took oaths as pharmacists to take care of the people we serve. In health care, there are limited dollars that can be spent, and our challenge is to make sure our resources are being used appropriately. By conducting this study, we attempted through all our possible means, to evaluate the overall impact of the edit on all our 12,000 members who use triptans. For those who suffered from frequent migraine episodes, we have the exception process in place to allow coverage for appropriately prescribed therapy.

We encourage you and others to continue to add to the body of knowledge regarding this disease and outcomes from various management programs. We thank you for your comments.

\section{Dear Dr. Barbuto,}

It was the dichotomy of outcomes related to medication use that you so poetically describe that was the impetus for our management program. The triptan edit was meant to promote the potential beneficial effects while minimizing the opportunity for negative outcomes, especially in our population, which demonstrated wide variations in usage patterns. We appreciate your comments and thank you for your insight.

Eric J. Culley, Pharm.D., D.U.R. Clinical Pharmacy Specialist,
Highmark Blue Cross Blue Shield
E-mail: eric.culley@highmark.com

Robert T. Wanovich, Manager, Clinical Services and Product Development, Adjunct Professor, Duquesne University Mylan School of Pharmacy

\section{REFERENCES}

1. Culley EJ and Wanovich RT. Medical and pharmacy cost and utilization outcomes of a quantity level limit on the 5-HTl agonists (triptans) by a managed care organization. J Man Care Pharm 2001;7(6):468-75

2. Zed J, Loewen PS, Robinson G. Medication-induced headache:overview and systematic review of therapeutic approaches. Ann Pharmacother 199;33:61-72

3. Stang PE, Osterhaus JT, Celentano DD. Migraine: patterns of healthcare use. Neurology 1994;44(suppl 4):S47-S54 